\title{
Thermal Imaging Device using Infrared-to-Visible Converter Made of Temperature Sensitive Phosphor
}

\author{
Takashiro Tsukamoto, Min Wang, and Shuji Tanaka \\ Department of Bioengineering and Robotics, Division of Mechanical Engineering, \\ Tohoku University, 6-6-01 Aramaki aza Aoba, Aoba-ku, Sendai, Miyagi 980-8579, Japan
}

\begin{abstract}
An infrared thermal imaging device using infrared-to-visible convertor made of $\mathrm{Eu}(\mathrm{TTA})_{3}$ was developed and evaluated. A polymer based structure enabled high thermal isolation with simple structure, which enabled detection of low temperature object such as human. In addition, noise reduction digital methods using averaging filter and Kalman filter were proposed and evaluated. Both digital filters increased signal-to-noise ratio from 8.1 to 18 and 21, respectively, which means both filters had similar noise reduction effect. However, the Kalman filter had faster response compared with the averaging filter, therefore it is suitable for the noise reduction method of the thermal imaging device.
\end{abstract}

Keywords: Infrared Thermal imaging, Eu(TTA)3, Temperature sensitive phosphor, Kalman filter

\section{Introduction}

An infrared (IR) thermal imaging is one of the non-contact thermal measurement method which can measure the temperature distribution of the object without disturbance. The IR thermal imaging device detects an IR radiated from the object, of which the intensity is dependent on the surface temperature, know as Stefan-Boltzmann low. The IR thermal imaging devices are classified into two groups: a quantum and a thermal types.

The quantum type thermal imaging device counts the IR photons using photoelectric effect. They have quite high sensitivity and fast response, however should operate under cryogenic temperature. Therefore, recently, thermal type devices, which can be operated under room temperature, have been payed much attention for commercial applications. The thermal type device detects the temperature change of a thermally-isolated microstructure induced by an incoming IR. The conventional thermal type devices use electrical ways to detect the temperature change of the microstructure such as using a thermistor [1], a PN diode [2], a thermo-pile [3] and pyroelectric effect [4]. However, these devices should be fabricated on a read out integrated circuit (ROIC), which makes a fabrication difficult and thus the fabrication cost increases. In addition, these electrical measurement methods require metal wirings which increase the heat loss from the micrcostructure.

One of the candidates to solve these problems is using an optical method to measure the temperature change of the thermally isolated microstructure. A bi-material type optical read-out thermal imaging device is a well-known example of them [5]. However, long suspension beams are needed for both high thermal isolation and high sensitivity, which reduces the fill factor.

Recently, another optical type thermal imaging device based on IR-to-visible conversion was proposed [6-8], in which high thermal isolation with simple structure can be achieved by using a polymer as a structural material. In this paper, we demonstrated the human detection using the IR-to-visible converter array and proposed a signal processing method to reduce observation noise.

\section{Principle of the thermal imaging}

Figure 1 shows the working principle of the IR-to-visible converter. A microstructure made of an IR absorber, a reflector, a polymer structure and 
a temperature sensitive film was suspended by narrow polymer beams. The temperature sensitive film was made of Europium(III) thenoyltrifluoroacetonate $\left(\mathrm{Eu}(\mathrm{TTA})_{3}\right)$, which emits the visible luminescence under excitation of ultraviolet (UV) light. The intensity of the luminescence reduces with the temperature [9], thus the temperature can be measured as the intensity of visible light [10].

The IR radiated from the observation object was focused on the suspended microstructures using an IR lens made of $\mathrm{Ge}$, which induces the temperature change. The phosphor $\left(\mathrm{Eu}(\mathrm{TTA})_{3}\right)$ was excited by LEDs and the luminescence from them were captured by a CCD camera through an optical band-pass filter.

The temperature of sensing element at a position $(x, y)$ was obtained as,

$$
S^{-1} \sum_{i, j=-3}^{3} K_{i, j}\left(\frac{I(x+i, y+j)-I_{0}(x+i, y+j)}{I_{0}(x+i, y+j)}\right)
$$

where $T_{\text {raw }}, T_{0}, I, I_{0}$ and $S$ are the temperature with and without IR irradiation, observed visible light intensity with and without IR irradiation and sensitivity of the phosphor $\left(\operatorname{Eu}(\mathrm{TTA})_{3}\right)$, respectively. $K_{i, j}$ is a kernel of Gaussian filter defined as

$$
K_{i, j}=0.073269 \exp \left(-\left(i^{2}+j^{2}\right) / 4.5\right) .
$$

\section{Noise reduction methods}

The captured image contains large noise, mainly comes from the photon shot noise of the CCD due to the low luminescence intensity from $\mathrm{Eu}(\mathrm{TTA})_{3}$. To obtain a high quality thermal image, noise reduction method is required. In this paper, we used two digital noise reduction methods: using a simple averaging method and a Kalman filter.

The averaged temperature, $T_{\text {avg, }}$ at a time, $t$, was calculated as

$$
T_{\text {avg }}(x, y, t)=\frac{1}{N} \sum_{i=0}^{N} T_{\text {raw }}(x, y, t-i \Delta t),
$$

where $N$ is a number of data for averaging and $\Delta t$ is a time interval between capturing the image.

The procedure of Kalman filtering is illustrated in Fig. 2. A thermal model shown in Fig. 2(a) was used to establish a state space model. Thus the state equation and output equation are obtained as

$$
X(t+\Delta t)=A X(t)+B V(t)
$$

and

$$
Y(t)=C X(t)+W(t),
$$

where $X(t)$ is a state vector consisting of the temperature, $T$, and the input power of IR, $P_{\text {in }}$, as

$$
X(t)=\left[\begin{array}{c}
T(x, y, t) \\
P_{\text {in }}(x, y, t)
\end{array}\right],
$$

and $Y(t), V(t)$ and $W(t)$ are an output vector, system noise and observation noise, respectively. $A, B$ and $C$ are coefficient matrices defined as
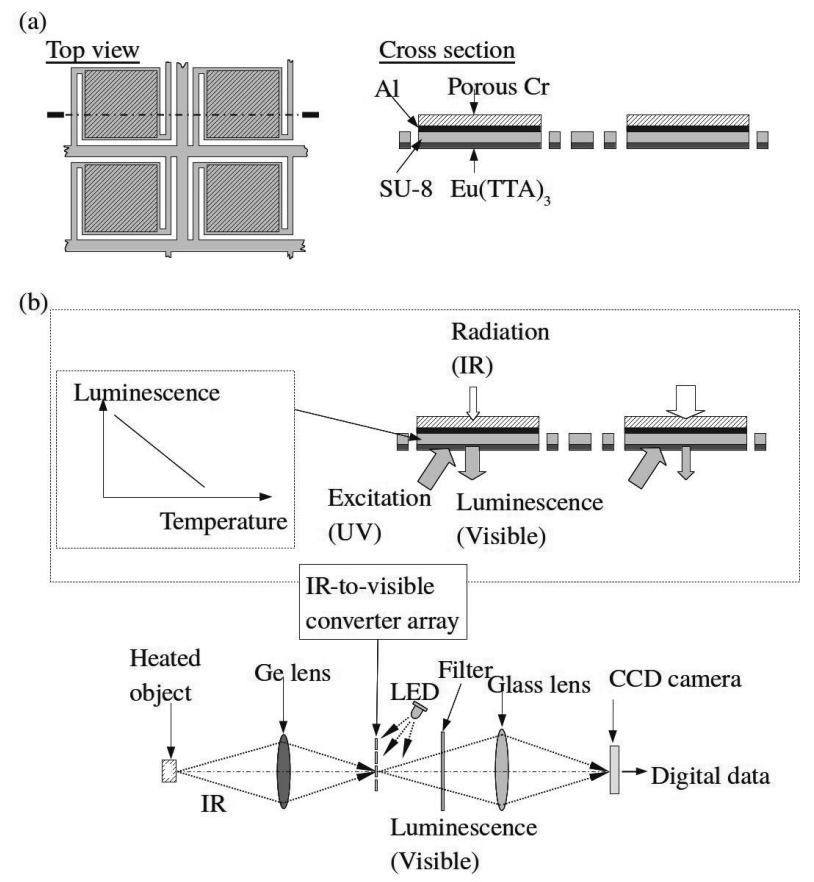

Figure 1. Working mechanism of the thermal imaging using IR-to-visible converter. (a) Structure of the IR-to-visible converter array and (b) principle of thermal imaging.

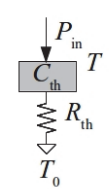

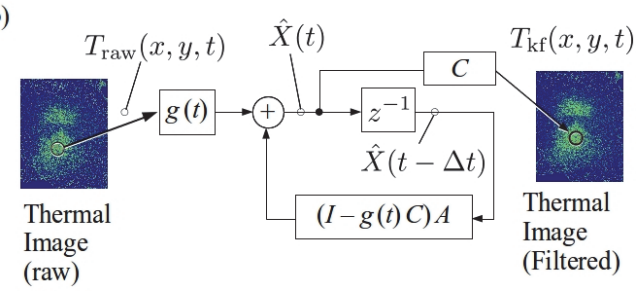

Figure 2. (a) Simplified thermal model of each sensing element and (b) signal flow of Kalman filter. 


$$
\begin{aligned}
& A=\left[\begin{array}{cc}
1-\frac{\Delta t}{R_{\mathrm{th}} C_{\mathrm{th}}} & \frac{\Delta t}{C_{\mathrm{th}}} \\
0 & 1
\end{array}\right], \\
& B=\left[\begin{array}{l}
1 \\
0
\end{array}\right]
\end{aligned}
$$

and

$$
C=\left[\begin{array}{ll}
1 & 0
\end{array}\right],
$$

respectively. The filtered output, $T_{\mathrm{kf}}$, was then calculated as

$$
T_{\mathrm{kf}}(x, y, t)=C \hat{X}(t),
$$

where $\hat{X}(t)$ is an estimated state vector calculated as,

$$
\hat{X}(t)=g(t) T_{\text {raw }}(x, y, t)+(I-g(t) C) A \hat{X}(t-1) .
$$

The Kalman gain, $g(t)$, and the covariance matrix, $P(t)$, can be calculated as

$$
g(t)=\frac{\left(A P(t-\Delta t) A^{T}+\sigma_{\mathrm{v}}^{2} B B^{T}\right) C^{T}}{C\left(A P(t-\Delta t) A^{T}+\sigma_{\mathrm{v}}^{2} B B^{T}\right) C^{T}+\sigma_{\mathrm{w}}^{2}}
$$

and

$$
P(t)=(I-G(t) C)\left(A P(t-\Delta t) A^{T}+\sigma_{v}^{2} B B^{T}\right),
$$

where $\sigma_{\mathrm{v}}$ and $\sigma_{\mathrm{w}}$ are the standard deviation of system noise and observation noise, and $I$ is a unit matrix, respectively.

\section{Device fabrication}

Figure 3 shows the fabrication process of the test device. The detailed fabrication process was described in the other paper [8]. First thin film of $\mathrm{SiO}_{2}$ was thermally grown on the $\mathrm{Si}$ substrate, and the SU-8 was patterned on it. Then Al thin film and porous $\mathrm{Cr}$ were patterned on it to form a reflector and IR absorber, respectively. The Si substrate and $\mathrm{SiO}_{2}$ thin film underneath the sensor was etched away from backside. The $\mathrm{Eu}(\mathrm{TTA})_{3}$ thin film was then vacuum deposited on the SU-8 structure. Finally, Parylene-C was deposited on an entire surface of sensors.

\section{Thermal imaging result}

Figure 4 shows the schematic of measurement

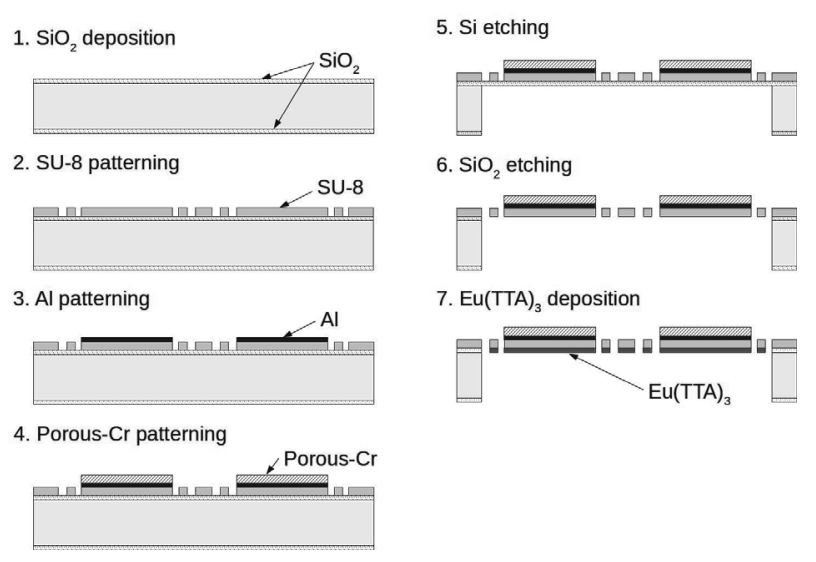

Figure 3. Fabrication process.

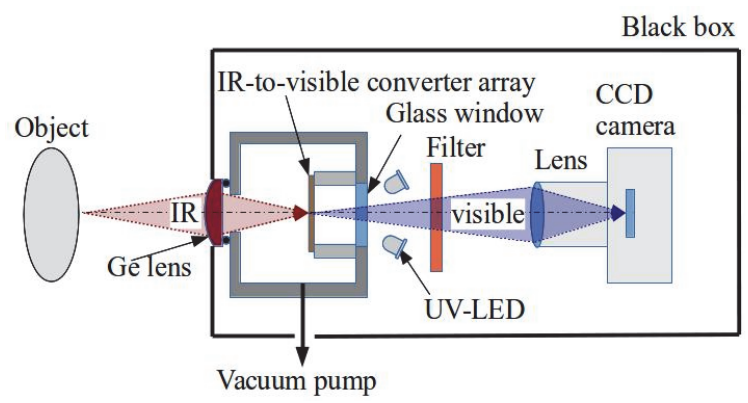

Figure 4. Experimental setup of thermal imaging using the fabricated IR-to-visible converter array.

system same as previous reported one [8]. The fabricated IR-to-visible converter array was placed in a vacuum chamber. The radiated IR was focused on the array using f/0.66 Ge lens. The exciting UV light was irradiated by using UV-LEDs (SSL-LXTO46355C, Lumex Inc., USA), and the luminescence was captured by a CCD camera (BU-50LN, Bitran Corp., Japan) through an optical band-pass filter $(607 \mathrm{~nm} \pm 21 \mathrm{~nm})$.

Figure 5 shows thermal images of human wearing glasses obtained by using Eqs. (1), (3) and (10). From these images, it is ovbious that both the averaging method and the Kalman filter could successfully reduce the noise and made the image clear. Figure 6 shows the time response of each thermal image corresponding to the center of face. The signal-to-noise ratios calculated from Fig. 6 were 8.1, 18 and 20 for raw, averaged and Kalman filtered results, respectively, which indicates both the averaging method and the Kalman filter had similar effect to reduce the noise. However, the averaging method induced large delay compared with the Kalman filter. Therefore, the Kalman filter was considered as better method to reduce the noise of the proposed thermal imaging device. 


\section{Summary}

A thermal imaging device using IR-to-visible converter array made of temperature sensitive phosphor was developed and human detection was successfully demonstrated. Two types of noise reduction methods using simple averaging and Kalman filter were proposed and evaluated. Both the Kalman filter and averaging method had similar noise reduction performance, however, the Kalman filter had small delay compared with the averaging method.

\section{Acknowledgement}

This work was partly supported by Creation of Innovation Centers for Advanced Interdisciplinary Research Areas Program.

\section{References}

1. R. A. Wood, C. J. Han and P. W. Kruse, "Integrated uncooled infrared imaging arrays", Proc. IEEE Solid State Sensor and Actuator Workshop, pp. 132-135 (1992).

2. T. Ishikawa, M. Ueno, K. Endo, Y. Nakaki, H. Hata, T. Sone, M. Kimata and T. Ozeki, "Low-cost 320x240 uncooled IRFPA using a conventional silicon IC process", Proc. SPIE, 3698 (1999) 556.

3. T. Kanno, M. Sage, S. Matsumoto, M.Uchida, N. Tsukamoto, A. Tanaka, S. Itho, A. Nakazato, T. Endoh, S. Tohyama, Y. Yamamoto, S. Murashima, M. Fujimoto and N. Teranishi, "Uncooled infrared focal plane array having 128X128 thermopile detector elements", Proc. SPIE, 2269 (1994) 450.

4. C. M. Hanson, H. Beratan, R. Owen, M. Corbin and S. McKenney, "Uncooled thermal imaging at Texas Instruments", Proc. SPIE, 1735 (1992) 17.

5. Y. Zhao, M. Mao, R. Horowitz, A. Majumdar, J. Varesi, P. Norton and J. Kitching, "Optomechanical uncooled infrared imaging system: design, microfabrication, and performance", J. Microelectromech. Syst., 11 (2002) 136.

6. M. Alfaro, G. Paez and M. Strojnik, "Conversion of absorbed thermal radiation into visible using europium thenoyltrifluoroacetonate", Appl. Opt., 49 (2010) 5444.

7. M. Wang, T. Tsukamoto and S. Tanaka, "Infrared thermal detector array using Eu(TTA) (a)

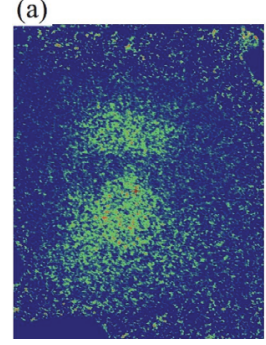

(b)

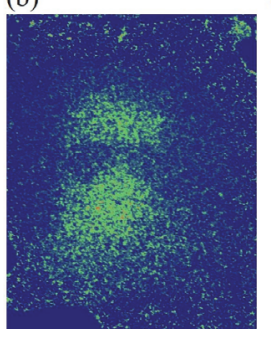

(c)

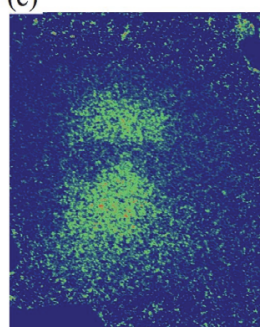

Figure 5. Obtained thermal image of human face wearing glasses. (a) As calculated thermal image (Eq. (1)), and after applying (b) an averaging filter (Eq. (3)) and (c) a Kalman filter (Eq. (10)).

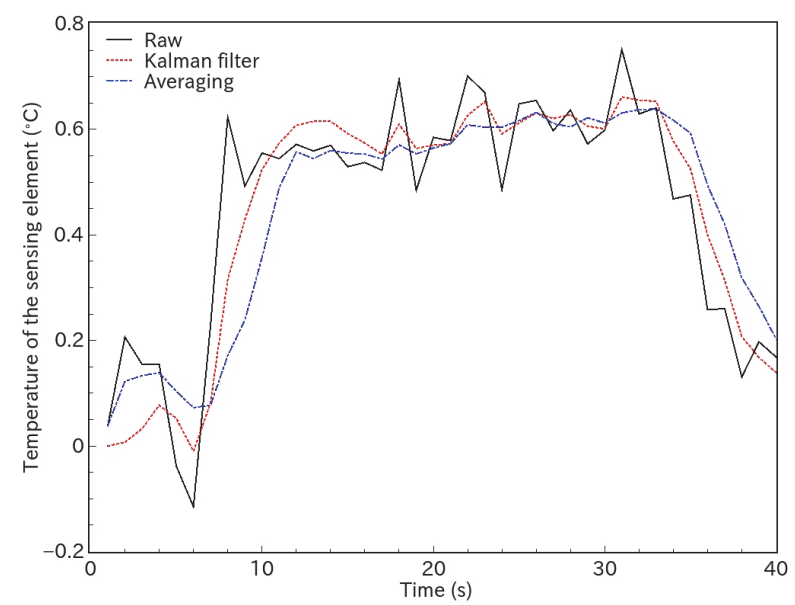

Figure 6. Time response of the sensor output. A solid line, a dotted line and a dashed line show an as calculated response, a Kalman filtered result and an averaged result, respectively.

3-based temperature sensitive paint for optical readable thermal imaging device", $J$. Micromech. Microeng., 25 (2015) 035012.

8. M. Wang, T. Tsukamoto and S. Tanaka, "Uncooled infrared thermal imaging sensor using vacuum-evaporated europium phosphor", J. Micromech. Microeng., 25 (2015) 085001.

9. M. L. Bhaumik, "Quenching and Temperature Dependence of Fluorescence in Rare-Earth Chelates", J. Chem. Phys., 40 (1964) 3711.

10. P. Kolodner and J. A. Tyson, "Microscopic fluorescent imaging of surface temperature profiles with $0.01^{\circ} \mathrm{C}$ resolution", Appl. Phys. Lett., 40 (1982) 782. 